\title{
Errores en el diagnóstico de apendicitis aguda en ancianos. Estudio de un caso clínico y revisión de la literatura
}

\author{
Diagnostic errors in acute appendicitis in the elderly. Study of a clinical case \\ and revision of the literature
}

Leopoldo Herrera Chabert, ${ }^{*}$ Jacobo Joffe Fraind,* Luis Enrique Llamas Prieto*

\begin{abstract}
Palabras clave: Apendicitis del anciano, error en el diagnóstico, diagnóstico oportuno y pronta cirugía.
\end{abstract}

Key words: Appendicitis in the aged, difficult diagnosis, early diagnosis and surgical treatment.

* Departamento de Gastrocirugía. Hospital San Javier. Guadalajara, Jalisco, México.

Recibido: 06/04/2016 Aceptado: 30/11/2016

\section{RESUMEN}

Introducción: Por más de seis décadas, la apendicitis aguda en pacientes longevos continúa teniendo errores diagnósticos, lo que resulta en la demora de un diagnóstico oportuno y un retraso en la cirugía. La apendicitis del anciano se caracteriza por un retardo en el diagnóstico y tratamiento de índole multifactorial. El hallazgo habitual durante la cirugía es un apéndice perforado con peritonitis regional o generalizada. La muerte en estos individuos es secundaria a sepsis abdominal por siembra bacteriana de origen intestinal. El curso postoperatorio en un anciano con un proceso inflamatorio mínimo en el apéndice cecal, como el que se describe, es habitualmente benigno al controlarse oportunamente la contaminación peritoneal. $\mathrm{Si}$ una comorbilidad no interviene en el postoperatorio, el egreso hospitalario es rápido. Caso clínico: En el caso que se presenta, el apéndice tuvo un aspecto normal, como se muestra en la fotografía. Nosotros decidimos resecarlo por ausencia de otras anormalidades en la cavidad abdominal. En el estudio histopatológico se describieron cambios inflamatorios confinados a mucosa y submucosa. No hubo perforación. Estas modificaciones pudieron ser secundarias a una severa hipoperfusión por el estado de la mujer. Conclusiones: Resulta peligroso concluir que "aunque macroscópicamente se aprecie normal el apéndice cecal en sujetos longevos, sería muy razonable extirparlo" para evitar un desenlace como el del caso que se presenta.

\section{ABSTRACT}

Introduction: For more than six decades, acute appendicitis in the aged remains a difficult diagnosis that results in a delay in an appropriate treatment. Appendicitis in the elderly is characterized by a delay in the diagnosis and treatment of multifactorial nature. The usual finding during surgery is an appendix with regional or widespread peritonitis. Death in these patients is secondary to abdominal sepsis by bacterial seeding of intestinal origin. The postoperative course in an old person with a minimal inflammatory process in the cecal appendix, such as the one described in this case, is usually benign if there is an appropriate control of peritoneal contamination. If comorbidity does not intervene in the postoperative period, hospital discharge is rapid. Case report: In the case that is presented, the cecal appendix was normal, as shown in the picture. We decided to resect it because of the absence of other abnormalities in the abdominal cavity. The histopathological study described inflammatory changes confined to the mucosa and submucosa. There was no perforation. These changes might have been secondary to a severe hypoperfusion due to the state of the patient. Conclusions: It is dangerous to conclude that "although the cecal appendix in older patients might seem normal, it would be very reasonable to remove it" to avoid an outcome like that of the case presented.

\section{INTRODUCCIÓN}

$\mathrm{H}$ asta la fecha, continúa siendo innegable que la apendicitis aguda en ancianos (llamada así para los grupos poblacionales con más de 60 años de edad) sigue siendo un enigma, ya que por más de seis décadas no ha tenido resultados satisfactorios; es decir, no ha habido cambios concretos en cuanto a un avance convincente en el tratamiento quirúrgico de manera oportuna, ya que continúa con una alta incidencia de complicaciones vinculada a errores en el diagnóstico, demora en acudir al servicio de urgencias y retardo en la cirugía, lo que se manifiesta con alta incidencia de perforaciones, abscesos y alta mortandad, como se aprecia en los cuadros I y II. 
Cuadro I. Duración del retraso relacionado a apendicectomías en 61 pacientes $>65$ años.

\begin{tabular}{lcccc} 
Intervalo & $\begin{array}{c}<\mathbf{2 4} \mathbf{h} \\
\text { No } \mathbf{\%})\end{array}$ & $\begin{array}{c}\mathbf{2 4 - 7 2} \mathbf{h} \\
\text { No (\%) }\end{array}$ & $\begin{array}{c}\mathbf{3 - 7} \text { días } \\
\text { No (\%) }\end{array}$ & $\begin{array}{c}\mathbf{7} \text { días } \\
\text { No (\%) }\end{array}$ \\
\hline Del inicio del dolor hasta la hospitalización & $30(49)$ & $21(34)$ & $9(15)$ & $1(2)$ \\
De la admisión hasta la cirugía & $55(90)$ & $3(5)$ & $2(3)$ & $1(2)$ \\
Del inicio del dolor hasta la cirugía & $19(31)$ & $30(49)$ & $8(13)$ & $3(5)$
\end{tabular}

Cuadro II. Sumario de la literatura de apendicectomías en individuos mayores de 60 años de edad.

\begin{tabular}{|c|c|c|c|c|c|c|c|}
\hline Estudio & Año & Edad & $\mathbf{N}$ & $\begin{array}{c}\text { Perforación y } \\
\text { absceso } \\
\%\end{array}$ & $\begin{array}{c}\text { Error Dx. } \\
\quad \%\end{array}$ & $\begin{array}{c}\text { Complicación } \\
\%\end{array}$ & $\begin{array}{c}\text { Mortalidad } \\
\%\end{array}$ \\
\hline Wolff y Hindman ${ }^{28}$ & 1945 & 88 & - & 58 & 25 & 41 & 4.5 \\
\hline Goldenberg 29 & 1949 & $>60$ & 129 & 66 & 11 & 25 & 4.6 \\
\hline Hubbell, et al ${ }^{30}$ & 1956 & $>50$ & 100 & 49 & 16 & 36 & 7 \\
\hline Coran y Wheeler ${ }^{31}$ & 1959 & $>60$ & 28 & 67 & 36 & 41 & 7.1 \\
\hline Albano, et a $b^{32}$ & 1961 & $>60$ & 93 & 38 & 20 & 61 & 20 \\
\hline Freund, et $a l^{33}$ & 1967 & $>60$ & 77 & 62 & 5 & 48 & 1.3 \\
\hline Owens y Hamit ${ }^{34}$ & 1970 & $>80$ & 13 & 77 & - & 23 & 23 \\
\hline Blake y Lynn ${ }^{35}$ & 1972 & $>75$ & 34 & - & - & - & 11.8 \\
\hline Pieper, et a ${ }^{36}$ & 1974 & $>60$ & 42 & 57 & 33 & 73 & 4.7 \\
\hline Horattas, et $a l^{37}$ & 1983 & $>60$ & 96 & 72 & 30 & 32 & 4.2 \\
\hline Luckmann $^{38}$ & 1984 & $>80$ & 193 & 75 & 7 & - & 4.7 \\
\hline Paajanen, et $a l^{39}$ & 1988 & $>80$ & 100 & 49 & 31 & 40 & 7 \\
\hline Franz, et al ${ }^{40}$ & 1990 & $50-70$ & 46 & 93 & 30 & - & 4.3 \\
\hline Ronen Rub MD ${ }^{41}$ & 1993 & $>60$ & 61 & 52 & 5 & 41 & 3.3 \\
\hline
\end{tabular}

\section{HISTORIA}

El curso y desarrollo de la apendicitis aguda en pacientes ancianos difieren definitivamente del resto de la población en general, ya que se caracterizan por una mayor incidencia de perforaciones $y$, en consecuencia, un aumento de la morbilidad y mortalidad, ya sea por una presentación tardía o atípica, errores diagnósticos o una subyacente diferencia patológica en el órgano afectado que se investiga.

Se estima que uno de cada 15 individuos de la población en general desarrollará apendicitis aguda en el transcurso de su vida. ${ }^{1}$ Aunque la incidencia máxima de este padecimiento ocurre entre los 15 y 24 años de edad, ${ }^{2-5}$ del 5 al $10 \%$ de todas las apendicitis agudas tienen lugar en personas mayores de 60 años: ${ }^{3-5}$ esto representa el $5 \%$ de todas las condiciones abdominales agudas en esta etapa de la vida. ${ }^{5}$ Sin embargo, la incidencia de apendicitis en los individuos longevos parece aumentar con el incremento de la expectativa de vida.

Reginald Fitz describió por primera vez esta condición en 1886; la mortalidad de la apendicitis aguda se reportaba del $40 \% .{ }^{6}$ Con la introducción de la anestesia general, técnicas antisépticas de Joseph Lister en 1870 y la disponibilidad de sulfamidas, la tasa de mortalidad registrada disminuyó significativamente, y en 1944 fue simplemente 2.4\%. ${ }^{7}$ Tras el descubrimiento del Bacteroides fragilis dentro del apéndice y en heridas infectadas por apendicectomía por Leigh en $1974,{ }^{8}$ el uso generalizado de los antibióticos contra los organismos 
anaerobios redujo aún más la incidencia de sepsis intraabdominal e infección de heridas quirúrgicas, con lo que se logró una subsecuente mejoría en la tasa de mortalidad, entre 0.8 y $1.6 \%{ }^{7-9}$

Para fines de investigación, las personas de edad avanzada se dividen en tres categorías:

1. El viejo joven (edad de 65 a 74 años)

2. El viejo medio (edad de 75 a 79)

3. El viejo mayor (mayores de 80 )

Los adultos mayores son grupos poblacionales que tienen 65 años de edad en adelante y son quienes representan el segmento de más rápido crecimiento en la población de los Estados Unidos, por lo que son los que más se examinan en el departamento de emergencia (DE). ${ }^{10}$ Según algunas estimaciones, los adultos longevos representarán una cuarta parte de las visitas en el DE en los Estados Unidos para el año 2030. ${ }^{11}$

\section{Otros datos de interés relacionados con enfermedades abdominales en ancianos}

El dolor abdominal es la queja más común que se presenta en el DE en adultos de la tercera edad; por otra parte, en todas las edades, el dolor abdominal es la cuarta queja principal más común en el DE. ${ }^{12}$

Los retos para diagnosticar y manejar el dolor abdominal en los adultos de edad avanzada son multifactoriales; esto se debe a que hay falta de hallazgos clínicos evidentes y diferentes presentaciones de los trastornos intraabdominales. En un estudio comparativo entre personas jóvenes y mayores, médicos especialistas en medicina de emergencia encontraron que los sujetos viejos de manera consistente son los más complejos para diagnosticar y los más difíciles de evaluar, ya que requieren más tiempo y recursos para ser diagnosticados y tratados. ${ }^{13}$

Las causas más comunes de cirugías de emergencia en el grupo de edad $>65$ años son la obstrucción mecánica del intestino (45\%), perforación (18\%) y hernia encarcelada (18\%). ${ }^{14}$ La obstrucción del intestino grueso en las personas de la tercera edad es causada generalmente por malignidad y, ocasionalmente, por vólvulos de sigmoides. ${ }^{15}$ La obstrucción del intestino delgado casi siempre es causada por adherencias, inde- pendientemente de la edad del paciente. Las hernias de la pared abdominal aumentan en la vejez debido a la mayor laxitud de los tejidos. Por otra parte, hasta $30 \%$ de las personas de mayor edad que se presentan al servicio de urgencias con dolor abdominal tiene colecistitis. ${ }^{15}$

Las tasas de apendicitis perforada, complicaciones postoperatorias y mayor mortalidad pueden estar relacionadas de manera directa con error en el diagnóstico, lapso desde el inicio de los síntomas hasta la admisión a urgencias e inicio de la cirugía.

\section{Cambios patofisiológicos en el adulto mayor que influyen en el retraso en el diagnóstico}

Estas modificaciones, secundarias al envejecimiento, causan una mayor susceptibilidad a enfermedades intraabdominales, así como presentaciones clínicas anormales o atípicas. Estos cambios ocurren desde las células hasta niveles sistémicos, especialmente en los sistemas inmunológico, genitourinario, gastrointestinal, cardiovascular y nervioso.

Inmunológico. Los viejos corren un riesgo más frecuente y severo de adquirir graves infecciones debido a que la inmunorregulación se encuentra alterada en los adultos longevos. El envejecimiento de las células B disminuye la capacidad de desarrollar inmunidad humoral (anticuerpos) para nuevas infecciones o antígenos, lo que aumenta el riesgo de infecciones. ${ }^{16}$ La respuesta de los linfocitos $T$ también cambia con el envejecimiento, lo que se manifiesta por una disminución de la cantidad y calidad de las células $T$ y una disminuida respuesta inmune a antígenos conocidos, posiblemente debido más a cambios en el fenotipo hacia las células T inmunosupresoras. ${ }^{17,18}$ Estos desórdenes tienen consecuencias para la interpretación de la cuenta de glóbulos blancos; sin embargo, por otra parte, un conteo bajo no excluye la inmunodeficiencia funcional. El envejecimiento se asocia con menor respuesta a pirógenos; por lo tanto, hay un descenso de la temperatura basal del cuerpo, cambios en la homeostasis térmica y una disminución en la producción y conservación de calor. La inmunorregulación afectada también tiene una consecuencia en la disminución de la vigilancia inmunológica humoral, la principal defensa del cuerpo contra el desarrollo de células cancerosas. 
Genitourinario. Cambios con el envejecimiento en los riñones incluyen reducción en el número de glomérulos y disminución de la función glomerular. Estas modificaciones son causadas por ambos efectos; daño a largo plazo de las comorbilidades existentes, tales como hipertensión arterial y diabetes, y alteración de las arteriolas aferentes y eferentes, que resulta en daño glomerular. La tasa de filtración glomerular desciende con la edad a partir de la cuarta década, y luego decrece por cerca de 8 $\mathrm{ml} / \mathrm{min}$ por década, lo que resulta en una baja en el aclaramiento de fármacos y metabolitos. ${ }^{19}$ Cambios en la membrana basal y el desarrollo de pequeños divertículos en los túbulos renales distales promueven la estasis urinaria y el crecimiento bacteriano. ${ }^{19} \mathrm{El}$ envejecimiento de los riñones también disminuye la capacidad de concentrar la orina, lo que hace que los adultos mayores sean más propensos a la deshidratación. Hormonalmente, los riñones de individuos de edad avanzada han reducido la producción de eritropoyetina, lo que induce al adulto mayor hacia la anemia de lentas pérdidas de sangre. ${ }^{19}$

Los efectos del envejecimiento en el sistema gastrointestinal (Gl) también predisponen a los sujetos con condiciones patológicas abdominales. El estómago tiene un tiempo de vaciamiento ligeramente enlentecido; de la misma manera, el acomodamiento del fondo gástrico se encuentra alterado, e igualmente puede aumentar la secreción ácida, secundario a la menor producción de prostaglandinas.

Hígado. El flujo sanguíneo del hígado y su masa hepática baja con el envejecimiento, lo que resulta en una disminuida síntesis de albúmina y reducción de la fase uno del metabolismo de medicamentos. El descenso en el metabolismo de la función del citocromo P450 puede ser aun mayor en los hombres viejos que en las mujeres mayores. ${ }^{20}$ En el colon, el número de divertículos en el intestino aumenta con la edad. A causa de anorexia fisiológica por envejecimiento, hay una menor ingesta de nutrientes y líquidos, la cual predispone al adulto mayor al estreñimiento, malnutrición y deshidratación. ${ }^{21,22}$

Ambos sistemas, el central y el nervioso periférico son afectados también por el envejecimiento. Aumenta la prevalencia de la demencia y el deterioro cognitivo en la senectud, lo que oculta los síntomas y enmascara la historia mé- dica. Periféricamente, el dolor y la sensación de la temperatura disminuyen a medida que la percepción del tipo de dolor en los nervios sensores cambia lentamente de fibras A delta (rápido, agudo y punzante) a una dependencia de fibras $\mathrm{C}$ (conductoras más lentas). ${ }^{23}$ Esta reducción en la sensación puede contribuir a la falta de signos peritoneales en muchos adultos mayores.

Además, muchos longevos están tomando fármacos antiinflamatorios no esteroideos (AINE). Estos medicamentos no sólo pueden causar enfermedades Gl y renales, sino que también disminuyen la respuesta febril. El uso crónico de esteroides también aumenta el riesgo de patología Gl, como enfermedad úlcero-péptica, y mitiga la respuesta inmune. Los $\beta$-bloqueadores inhiben la taquicardia asociada con fiebre, dolor o infecciones.

\section{IMPEDIMENTOS DIAGNÓSTICOS}

Entre las dificultades que ya fueron descritas anteriormente y que confunden y retrasan el diagnóstico de trastornos intraabdominales en los adultos mayores, todavía hay más: hay una historia de consumo de múltiples medicamentos y enfermedades preexistentes como diabetes mellitus, hipertensión arterial, insuficiencia cardiaca congestiva, insuficiencia renal, edema pulmonar, enfermedad pulmonar obstructiva crónica, osteoartritis degenerativa o secuelas de un accidente cerebrovascular, que ponen en riesgo a la persona, no sólo alterando las manifestaciones clásicas y evidentes, sino también ocasionando la imposibilidad de obtener una historia clínica precisa.

La alta mortalidad registrada en las personas ancianas que requieren cirugía de emergencia refleja la disminución de reserva física, demora en el diagnóstico y las comorbilidades agregadas vistas en estos grupos de edad. Esto claramente muestra que el riesgo de mortalidad y comorbilidad continúan creciendo conforme avanza el tiempo.

A menudo, la dificultad inicial en recibir asistencia clínica en personas mayores no es otra cosa que un retraso en la obtención de atención médica por parte del paciente. La disminución en la respuesta del sistema inmune, insensibilidad a la percepción del dolor, deterioro cognitivo, aislamiento social y factores familiares, religiosos y culturales son causas para contribuir a este retraso. Como resultado, los individuos ancianos se 
presentan en urgencias en una etapa avanzada, ostentando ya una patología que pone en peligro su vida. Esto se ilustra, por ejemplo, cuando hay ausencia de síntomas clásicos asociados con la enfermedad úlcero-péptica. En esta condición, el dolor y ardor epigástricos, náuseas y anorexia pueden estar ausentes en la persona vieja, y hasta un 30\% de las presentaciones con esta afección no tiene ningún dolor abdominal. ${ }^{24}$

Una historia de la ingesta de medicamentos es muy importante en personas sexagenarias o mayores. Por ejemplo, los corticosteroides, antihipertensivos, antiinflamatorios no esteroideos y la aspirina predisponen a la ulceración péptica y también enmascaran los síntomas de la enfermedad quirúrgica.

Condiciones médicas preexistentes como demencia o disfasia posterior a un accidente cerebrovascular o diabetes mellitus pueden quebrantar la capacidad del sujeto para dar una adecuada historia clínica y, como resultado, condiciones intraabdominales pueden cursar sin un diagnóstico preciso y oportuno. Los síntomas pueden ser vagos o subestimados, en donde características y signos evidentes del padecimiento necesitan ser sacados, buscados o encontrados para asegurar una evaluación adecuada y rápida.

Hay que tomar en cuenta que al examinar el abdomen en una persona mayor, los signos cardinales de dolor en abdomen, como resistencia muscular, distensión abdominal, ruidos intestinales ausentes o disminuidos, pueden no estar presentes. La pérdida de masa muscular, en particular de la pared abdominal, hace que la resistencia abdominal sea menos evidente.

También, hay que tener siempre en mente que todas las enfermedades médicas asociadas que presentan las personas de la tercera edad, más la ingesta de medicamentos y el aislamiento social pueden contribuir a una atención retardada en el diagnóstico y a una demora en el tratamiento quirúrgico de pacientes de este grupo de edad.

De la misma manera, presentaciones inusuales o atípicas de la enfermedad aumentan la prevalencia de un error diagnóstico y retraso en recibir tratamiento quirúrgico oportuno en individuos longevos; por ejemplo, una apendicitis aguda puede presentarse como colitis, indigestión o estreñimiento.

Por otra parte, en causas extraabdominales de dolor agudo, es necesario ser rigurosamente desconfiado $-\mathrm{y}$, por otra parte, insistente en encontrar la causa del dolor (por ejemplo, deben descartarse síndrome coronario agudo o neumonía basal).

La posibilidad de por vida de desarrollar apendicitis aguda es de 7\%, y esto disminuye con la edad; sin embargo, el crecimiento de la población de edad avanzada ha conducido a una mayor incidencia de apendicitis aguda en la persona mayor de 65 años de edad. ${ }^{25}$ Hay estudios que muestran que debido a una presentación atípica de la apendicitis, y a veces retardada en sujetos ancianos, cuando se evalúan aquéllos que se sometieron a cirugía de emergencia por apendicitis aguda, la proporción de ellos con casos de apendicitis perforadas aumenta significativamente en comparación con casos de apendicitis no perforadas (12.9\% versus $2.9 \%) .{ }^{25}$

\section{Factores familiares, sociales, religiosos y culturales también juegan un papel importante en el retraso del diagnóstico y demora de la cirugía}

Algunas personas mayores ven el hospital como un lugar para ir a morir; otras sienten que el departamento de emergencia es apropiado solamente para quienes tienen trastornos potencialmente mortales.

La mayoría de los ancianos que viven con un familiar sienten el deseo de "no molestar al doctor", "no molestar a la familia (al hijo, la hija, la nuera, la esposa o amigos)" y esperar a ver "cómo están o cómo evolucionan las cosas en un par de días".

Aún hay más: otros pueden ser reacios a visitar un servicio de urgencias.

Para otros adultos longevos, el escenario del cuidado de un malestar abdominal puede verse como una molestia menor, insignificante o pasajera $y$, sin embargo, puede tratarse de un padecimiento que pone en peligro su vida.

Es importante, también, entender las propias palabras de los adultos mayores; por ejemplo: "No me siento bien". Es mejor pedirles que describan con sus propias palabras lo que significa para ellos eso. Si refieren "incomodidad en el estómago o vientre", pueden haber usado ese término en lugar de "dolor" para referirse a un malestar. Igualmente, pueden usar palabras como "ardor", "molestia", "indigestión", "se me 
ha ido el hambre", "tengo náusea" o "me siento enfermo". Estos sinónimos de "dolor" pueden servir como sustitutos para el dolor abdominal y ayudar a acelerar una concentración dirigida al diagnóstico definitivo.

En las últimas dos décadas se han producido tres grandes avances tecnológicos en el diagnóstico y tratamiento de la apendicitis aguda. La tomografía axial computarizada contrastada (TACC) ha demostrado ser el método más sensible y específico para el diagnóstico de apendicitis aguda, con 91\% de sensibilidad; el otro, la ultrasonografía, es menos sensible (53\%); ${ }^{26,27}$ la cirugía laparoscópica tiene la ventaja potencial no sólo de agregarse en el diagnóstico, sino también de proporcionar una oportunidad para el tratamiento definitivo. En adición, el colon por enema contrastado tiene una sensibilidad de $81 \%$. Estas dos modalidades diagnósticas son útiles también en la determinación de muchas otras condiciones, como perforación de víscera hueca, diverticulitis, pancreatitis y colecistitis aguda.

Otro beneficio añadido al diagnóstico temprano del TAC es que se pueden facilitar menos opciones terapéuticas invasivas, como el drenaje percutáneo de colecciones intraabdominales; por ejemplo, de un absceso. Por lo tanto, la cirugía de emergencia puede evitarse en conjunto.

\section{CASO CLÍNICO}

Se trata de una paciente de 79 años de edad que fue admitida al hospital por dolor abdominal.

Su historia médica más relevante incluía hipertensión arterial, insuficiencia cardiaca congestiva, hipertrofia del ventrículo izquierdo, bloqueo de la rama izquierda, enfermedad pulmonar obstructiva crónica, hipotiroidismo y obesidad.

La mujer fue admitida dos semanas previas en tres distintos hospitales y se le realizaron tres diferentes TAC por la misma molestia en el abdomen. En su primera admisión, el 19 de diciembre de 2014, se le hizo una TAC abdominal que demostró sólo escaso líquido libre en corredera parietocólica y fosa iliaca derechas. El resto del estudio fue normal. Se le diagnosticó colitis no específica y se dio de alta. En la segunda admisión, en otro hospital, el 20 de diciembre de 2014, se le efectuó una segunda TAC abdominal, con los mismos hallazgos. La paciente se sometió a una lapa- roscopia exploradora, que fue reportada como normal. El apéndice cecal fue visualizado $y$, como macroscópicamente se apreció normal, se decidió dejarlo intacto; al segundo día, se le realizó una colonoscopia, la cual fue normal.

Tras varios días de continuar con dolor abdominal, la mujer visitó por tercera vez el departamento de urgencias en nuestro hospital, el 30 de diciembre de 2014.

A su llegada, presentaba delirio, desorientación y confusión; fasciculación de mandíbula, dedos y manos; edema generalizado (anasarca), ingurgitación yugular bilateral, hipertensión arterial, insuficiencia cardiaca congestiva, insuficiencia renal, insuficiencia hepática, insuficiencia cerebral, bloqueo de la rama izquierda, derrame pleural bilateral, atelectasia de lóbulo inferior derecho, hipotiroidismo, deshidratación, alteraciones electrolíticas y desnutrición.

En el examen físico de abdomen, la enferma manifestó dolor en el cuadrante inferior derecho, en el punto de McBurney; no presentaba rebote positivo, pero había resistencia muscular voluntaria, con signo de Rovsin negativo. No tenía fiebre ni distención abdominal, no masas, flemón o plastrón a la palpación. Los ruidos intestinales eran hiperactivos. La mujer fue evaluada por USG y TAC de abdomen, en donde se descartó diverticulitis, pancreatitis, trombosis mesentérica, úlcera perforada, obstrucción intestinal, así como hernia estrangulada; el apéndice cecal no fue visualizado.

Se valoró con un ecocardiograma, que mostró cardiopatía hipertensiva, hipertrofia leve del ventrículo izquierdo, válvula aórtica normal, válvula mitral: insuficiencia moderada, dilatación moderada de la cavidad derecha, válvula tricúspide: insuficiencia moderada, FE $47 \%$, HAP severa, $80 \mathrm{mmHg}$.

El conteo de glóbulos blancos mostró 20,300 células $/ \mathrm{ml}$, bandas $2 \%$, hemoglobina $10.8 \mathrm{~g} / \mathrm{dl}$, hematocrito $32.7 \%$, potasio 3.2 $\mathrm{mEq} / \mathrm{l}$, sodio $140 \mathrm{mEq} / \mathrm{l}$, glucosa $110 \mathrm{mg} / \mathrm{dl}$, creatinina $1.1 \mathrm{mg} / \mathrm{dl}$, urea $63 \mathrm{mg} / \mathrm{dl}$, amilasa 30 $\mathrm{mg} / \mathrm{dl}$, lipasa $53 \mathrm{mg} / \mathrm{dl}$. El examen general de orina dio positivo a infección de vías urinarias.

Exámenes complementarios: bilirrubina total $0.40 \mathrm{mg} / \mathrm{dl}$ y directa $0.10 \mathrm{mg} / \mathrm{dl} ;$ AST $43 \mathrm{U} / \mathrm{l}$, ALT 53 U/I, GGT 235 U/I, LDH 1.033 U/I, TSH 12.5 $\mu \mathrm{U} / \mathrm{ml}$, amoníaco $14 \mathrm{mg} / \mathrm{dl}, \mathrm{CK}-\mathrm{MB} 1.1 \mathrm{U} / \mathrm{L}$, 
ácido láctico 0.9 mg/dl, VSG 33 mm/h, PCR 394 $\mathrm{mg} / \mathrm{dl}$, procalcitonina $0.7 \mathrm{ng} / \mathrm{ml}$, BNP $1.730 \mathrm{pg} /$ $\mathrm{ml}$, albúmina $2.3 \mathrm{~g} / \mathrm{l}$, colesterol $97 \mathrm{mg} / \mathrm{dl}$, prealbúmina $0.100 \mathrm{~g} / \mathrm{dl}$, transferrina $112 \mathrm{mg} / \mathrm{dl}$, nitrógeno en orina $7.12 \mathrm{mg} / \mathrm{dl}$, ácido úrico $7.0 \mathrm{mg} / \mathrm{dl}$.

La paciente fue tratada, primero, conservadoramente por dos días para estabilizar su crítica condición; se hidrató y se cubrió con antibióticos. Posteriormente, debido a que persistía el dolor en el cuadrante inferior derecho, se decidió llevar a cabo laparoscopia diagnóstica el 03 de enero de 2015.

En el procedimiento laparoscópico, después de descartar otra posibilidad patológica intraabdominal -incluyendo divertículo de Meckel—, se decidió realizar apendicectomía.

Los resultados histopatológicos del apéndice fueron apendicitis aguda del anciano con necrosis de la mucosa y submucosa (Figuras 1 a 4).

La enferma estuvo en terapia intensiva por 38 días con varias complicaciones postoperatorias; entre ellas, insuficiencia cardiaca congestiva, insuficiencia respiratoria, insuficiencia renal, insuficiencia cerebral, insuficiencia hepática y desórdenes metabólicos y electrolíticos, que fueron tratados multidisciplinariamente por más de un mes en la $\mathrm{UCl}$.

La mujer fue dada de alta el día 08 de febrero de 2015 en buenas condiciones. Actualmente realiza sus actividades cotidianas normales.

Revisando las cifras publicadas y realizando una regresión del tiempo, hemos observado casi ninguna mejora en las tasas de perforación/ absceso, errores diagnósticos, complicaciones y mortalidad en individuos mayores de 60 años de edad, y sólo una leve disminución en la mortalidad de 1945 a 1993.

\section{DISCUSIÓN}

La apendicitis en personas de la tercera edad, como se mencionó anteriormente, continúa siendo un desafío. Aunque hemos perfeccionado métodos de diagnóstico en apendicitis aguda - tales como el uso de la TAC contrastada, la ultrasonografía de alta resolución, laboratorio computarizado, rápido y confiable, así como la laparoscopia exploradora por cirugía de mínima invasión-, estas tecnologías modernas no han podido ayudar a efectuar un diagnóstico más rápido y evitar el retardo de una laparos- copia exploradora para acceder sin prórrogas a un tratamiento definitivo.

Desafortunadamente, a pesar de los avances tecnológicos modernos, el diagnóstico temprano sigue siendo un reto, y persiste siendo un problema quirúrgico. Los sujetos ancianos continúan presentándose tardíamente al departamento de urgencias; asimismo, siguen teniendo presentaciones inusuales o atípicas. Ambos métodos de diagnóstico, tanto la TAC contrastada abdominal como la laparoscopia exploradora, han aumentado su uso considerablemente en todo el mundo; sin embargo, no se han visto diferencias alentadoras en la disminución de complicaciones en las apendicitis del anciano, reducción del tiempo entre el inicio de los síntomas y la admisión al hospital o entre la admisión a urgencias y el inicio de la cirugía, un descenso en los días de hospitalización, o la morbilidad y

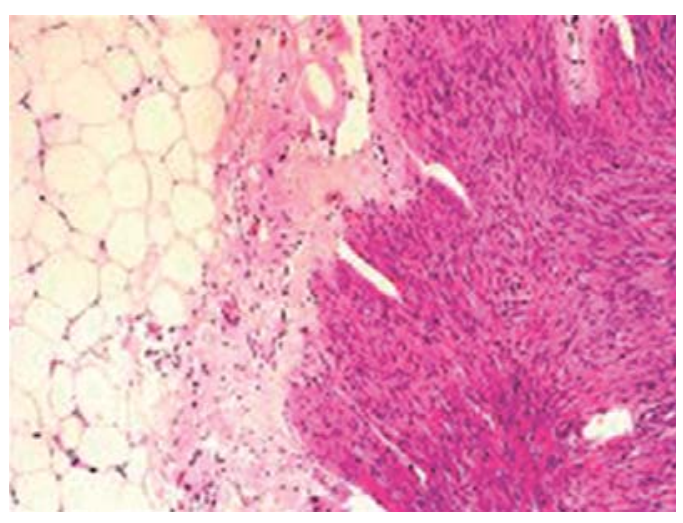

Figura 1. Histopatología; la pared muestra infiltrado polimorfonuclear que involucra la muscular.

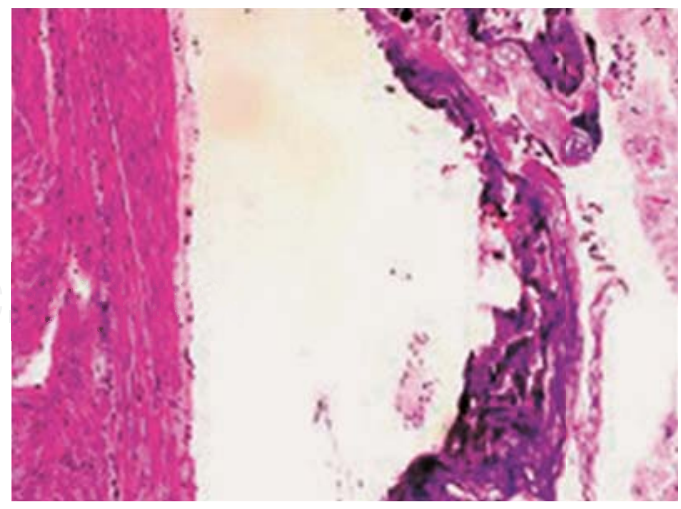

Figura 2. Histopatología; necrosis focal de mucosa y submucosa (apendicitis del anciano). 


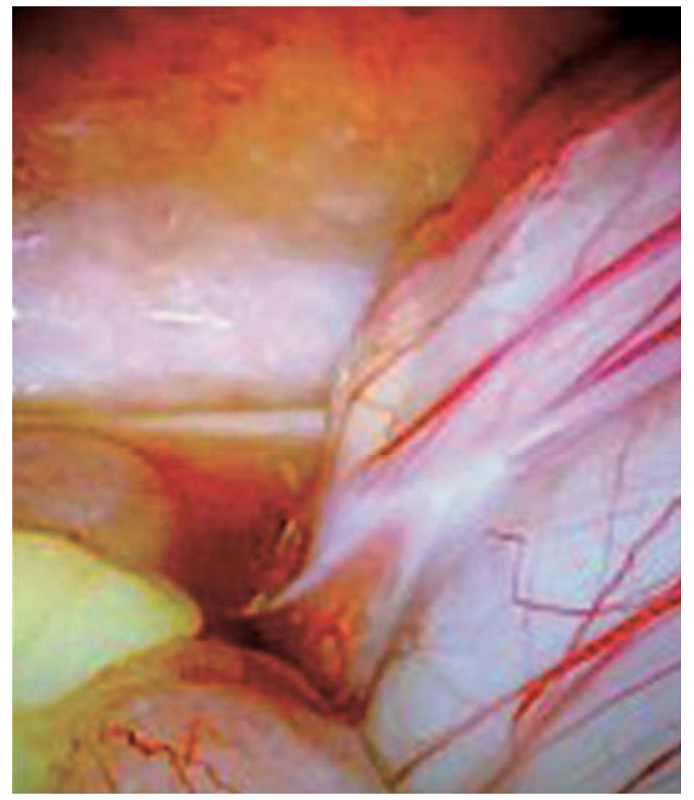

Figura 3. Exudado seroso en la fosa iliaca derecha.

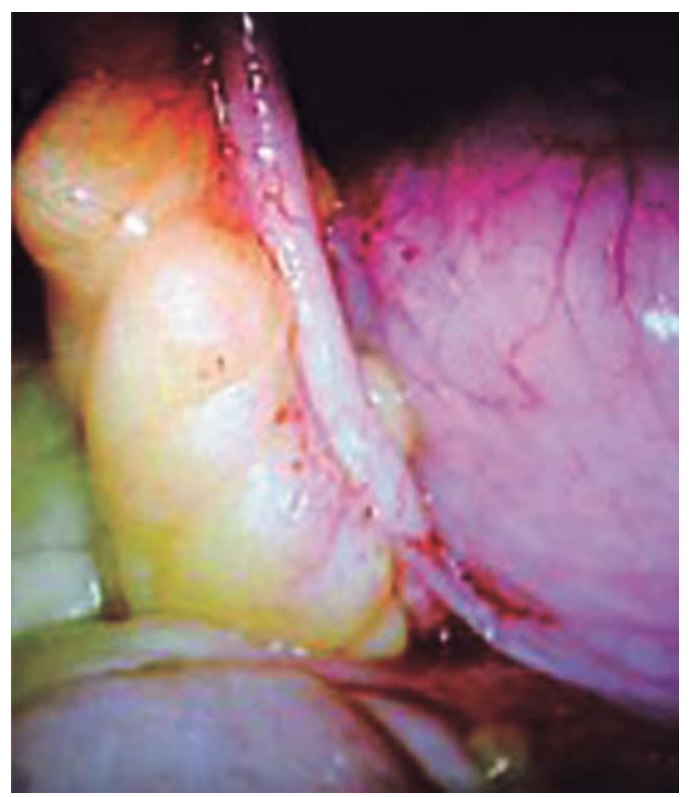

Figura 4. Apéndice cecal, diámetro y aspecto macroscópicamente normales. Observación macroscópica del apéndice opaca y demacrada y engrosamiento del mesoapéndice.

mortalidad, las cuales continúan hasta la fecha sin cambios significativos y alentadores.

Es muy importante tener siempre en mente que el dolor abdominal en pacientes de edad avanzada es un reto y seguirá siendo un desafío. También, hay que considerar el no dar mucha importancia al hecho de que el individuo no muestre un aumento en la cuenta de glóbulos blancos o que las placas simples de abdomen, el USG abdominal o incluso la TAC abdominal sean normales: uno debería ser perseverante en buscar otros signos, señales, evidencias -0 , inclusive, otros recursos-que nos lleven de la mano al diagnóstico oportuno de una catástrofe abdominal. Otras propuestas incluyen realizar diferentes estudios complementarios que rutinariamente no se realizan en personas jóvenes, como DHL, VSG, $\mathrm{PCR}$, ácido láctico, PFH, gasometría arterial, CK $\mathrm{MB}$, troponina y procalcitonina.

\section{CONCLUSIONES}

Finalmente, los resultados podrían mejorar considerablemente con una oportuna consideración en el diagnóstico de sujetos ancianos con dolor abdominal, la realización de exámenes complementarios que rutinariamente no se solicitan en apendicitis aguda del paciente joven, seguida de una rápida consulta quirúrgica; así mismo, durante la laparoscopia exploratoria diagnóstica, aunque macroscópicamente se aprecie normal el apéndice cecal, sería muy razonable extirparlo, porque hay una incidencia alta de apéndices que resultan estar atróficos en individuos ancianos. Sin embargo, hay otros signos indirectos de apendicitis aguda que hay que tomar en consideración y buscar, como engrosamiento del mesoapéndice, exudado libre en el área periapendicular, corredera parietocólica o fosa iliaca derecha; ganglios mesentéricos en íleon terminal; más aún, si la exploración laparoscópica del resto del abdomen fue negativa a otras patologías comunes de personas ancianas con dolor abdominal bajo. De este modo, con el tiempo, se podría lograr que muchos pacientes longevos volvieran a un buen nivel de su estado funcional en corto plazo, siempre y cuando el diagnóstico sea manejado con prontitud y el individuo de la tercera edad se someta al tratamiento quirúrgico en el momento de la presentación inicial del dolor abdominal.

\section{REFERENCIAS}

1. Peltokallio P, Jauhiainen K. Acute appendicitis in the aged patient. Study of 300 cases after the age of 60 . Arch Surg. 1970; 100: 140-143. 
2. Søreide O. Appendicitis - a study of incidence, death rates and consumption of hospital resources. Postgrad Med J. 1984; 60: 341-345.

3. Horattas MC, Haught R. Managing appendicitis in the elderly patient. AORN J. 1992; 55: 1282-1285.

4. Horattas MC, Guyton DP, Wu D. A reappraisal of appendicitis in the elderly. Am J Surg. 1990; 160: 282-285.

5. Franz MG, Norman J, Fabri PJ. Increased morbidity of appendicitis with advancing age. Am Surg. 1995; 61 : 40-44.

6. Peltokallio $\mathrm{P}, \mathrm{Tykkä} \mathrm{H}$. Evolution of the age distribution and mortality of acute appendicitis. Arch Surg. 1981; 116: 153-156.

7. Lau WY, Fan ST, Yiu TF, Chu KW, Lee JM. Acute appendicitis in the elderly. Surg Gynecol Obstet. 1985; 161: 157-160.

8. Leigh DA, Simmons K, Norman E. Bacterial flora of the appendix fossa in appendicitis and postoperative wound infection. J Clin Pathol. 1974; 27: 997-1000.

9. Smithy WB, Wexner SD, Dailey TH. The diagnosis and treatment of acute appendicitis in the aged. Dis Colon Rectum. 1986; 29: 170-173.

10. McCaig LF, Nawar EN. National hospital ambulatory medical care survey: 2004 emergency department summary. Advance date from vital and health statistics. Adv Data. 2006; 372: 1-29.

11. Wilber ST, Gerson LW, Terrell KM, Carpenter CR, Shah $\mathrm{MN}$, Heard K, et al. Geriatric emergency medicine and the 2006 Institute of Medicine reports from the Committee on the Future of Emergency Care in the U.S. health system. Acad Emerg Med. 2006; 13: 1345-1351.

12. Wofford JL, Schwartz E, Timerding BL, Folmar S, Ellis $\mathrm{SD}$, Messick $\mathrm{CH}$. Emergency department utilization by the elderly: analysis of the National Hospital Ambulatory Medical Care Survey. Acad Emerg Med. 1996; 3: 694-699.

13. McNamara RM, Rousseau E, Sanders AB. Geriatric emergency medicine: a survey of practicing emergency physicians. Ann Emerg Med. 1992; 212: 796-801.

14. Coroş MF, Copotoiu C, Copotoiu S, Ioniţă S, Dobre A, Sorlea S, et al. Surgical emergencies in elderly. Chirurgia (Bucur). 2008; 103: 283-290.

15. De Dombal FT. Acute abdominal pain in the elderly. J Clin Gastroenterol. 1994; 19: 331-335.

16. Ongrádi J, Stercz B, Kövesdi V, Vértes L. Immunosenescence and vaccination of the elderly II. New strategies to restore age-related immune impairment. Acta Microbiol Immunol Hung. 2009; 56: 301-312.

17. Cusi MG, Martorelli B, Di Genova G, Terrosi C, Campoccia G, Correale P. Age related changes in T cell mediated immune response and effector memory to Respiratory Syncytial Virus (RSV) in healthy subjects. Immun Ageing. 2010; 7: 14.

18. Grubeck-Loebenstein B. Fading immune protection in old age: vaccination in the elderly. J Comp Pathol. 2010; 142 Suppl 1: S116-S119.

19. Zhou XJ, Saxena R, Liu Z, Vaziri ND, Silva FG. Renal senescence in 2008: progress and challenges. Int Urol Nephrol. 2008; 40: 823-839.

20. Cotreau MM, von Moltke LL, Greenblatt DJ. The influence of age and sex on the clearance of cytochrome P450 3A substrates. Clin Pharmacokinet. 2005; 44: 33-60.
21. Radley S, Keighley MR, Radley SC, Mann CH. Bowel dysfunction following hysterectomy. Br J Obstet Gynaecol. 1999; 106: 1120-1125.

22. Bhutto A, Morley JE. The clinical significance of gastrointestinal changes with aging. Curr Opin Clin Nutr Metab Care. 2008; 11: 651-660.

23. Wickremaratchi MM, Llewelyn JG. Effects of ageing on touch. Postgrad Med J. 2006; 82: 301-304.

24. Hilton D, Iman N, Burke GJ, Moore A, O'Mara G, Signorini $\mathrm{D}$, et al. Absence of abdominal pain in older persons with endoscopic ulcers: a prospective study. Am J Gastroenterol. 2001; 96: 380-384.

25. Kamin RA, Nowicki TA, Courtney DS, Powers RD. Pearls and pitfalls in the emergency department evaluation of abdominal pain. Emerg Med Clin North Am. 2003; 21: 61-72, vi.

26. Stroman DL, Bayouth CV, Kuhn JA, Westmoreland $M$, Jones RC, Fisher TL, et al. The role of computed tomography in the diagnosis of acute appendicitis. Am J Surg. 1999; 178: 485-489.

27. Rao PM, Rhea JT, Novelline RA, Mostafavi AA, McCabe CJ. Effect of computed tomography of the appendix on treatment of patients and use of hospital resources. N Engl J Med. 1998; 338: 141-146.

28. Wolff WL, Hindman R. Acute appendicitis in the aged. Br J Sur. 1976; 63: 956-960.

29. Goldenberg IS. Acute appendicitis in the aged. Geriatrics. 1955; 10: 324-327.

30. Hubbell DS, Barton WK, Solomon OD. Appendicitis in older people. Surg Gynecol Obstet. 1960; 110: 289-292.

31. Coran AG, Wheeler HB. Early perforation in appendicitis after age 60. JAMA. 1966; 197: 745-748.

32. Albano WA, Zielinski CM, Organ $\mathrm{CH}$. Is appendicitis in the aged really different? Geriatrics. 1975; 30: 81-88.

33. Freund HR, Rubinstein E. Appendicitis in the aged. Is it really different? Am Surg. 1984; 50: 573-576.

34. Owens BJ, Hamit HF. Appendicitis in the elderly. Ann Surg. 1978; 187: 392-396.

35. Blake R, Lynn J. Emergency abdominal surgery in the aged. Br J Surg. 1976; 63: 956-960.

36. Pieper R, Kager L, Näsman P. Acute appendicitis: a clinical study of 1018 cases of emergency appendectomy. Acta Chir Scand. 1982; 148: 51-62.

37. Horattas MC, Guyton DP, Wu D. A reappraisal of appendicitis in the elderly. Am J Surg. 1990; 160: 291-293.

38. Lukmann R. Incidence and case fatality rates for acute appendicitis in California. A population-base study of effects of age. Am J Epidermiol. 1989; 129: 905-918.

39. Paajanen H, Kettunen J, Kostianen S. Emergency appendicitis in patients over 80 years old. Am Surg. 1994; 60: 950-953.

40. Franz MG, Norman J, Fabri PJ. Increased morbidity of appendicitis with advancing age. Am Surg. 1995; 61: 40-44.

41. Rub R, Margel D, Soffer D, Kluger Y. Appendicitis in the elderly: what has changed? Isr Med Assoc J. 2000; 2: $220-223$

Correspondencia:

Dr. Leopoldo Herrera Chabert

Tarascos Núm. 3473-810 Fracc. Monraz,

Guadalajara, Jalisco, 44670, México.

Tel: 52 (33) 36486236; (33) 14575882

E-mail: doctorchabert@hotmail.com 